\author{
Proceedings of the $9^{\text {th }}$ International Conference on Applied Informatics \\ Eger, Hungary, January 29-February 1, 2014. Vol. 2. pp. 231-243 \\ doi: 10.14794/ICAI.9.2014.2.231
}

\title{
Why do they always win?*
}

\section{Tamás László Balogh, János Kormos}

\author{
University of Debrecen \\ tamas.balogh@econ.unideb.hu, kormos.janos@inf.unideb.hu
}

\begin{abstract}
Several behavioral game theory models aim at explaining why "smarter" people win more frequently in simultaneous zero-sum games, a phenomenon, which is not explained by the Nash equilibrium concept. This type game theoretic models can be applied also in infocommunication problems. We use a computational model and a numerical simulation based on Markov chains to describe player behavior and predict payoffs.
\end{abstract}

Keywords: computational game theory, non-cooperative games, outguessing, Markov chains, reasoning, communication networks

MSC: 60J20, 62P20, 68U20, 91A10

\section{Introduction}

Since the birth of experimental economics, thousands of experiments have been conducted to observe the behavior of decision makers in different situations (see e.g. [5]).

However, the most famous equilibrium concept - the Nash equilibrium [15] - has proved to be unable to explain the outcome of several game theoretical experiments, predicting that human thinking is more complicated than pure rationality.

In this paper we investigate simultaneous, two-person, zero-sum, repeated games that do not have a pure strategy Nash equilibrium and the players' decisions depend only on their actions in the previous round of the game. Here, the stochastic processes of the players' decisions and their expected payoffs can be described by Markov chains.

We give a brief survey about the application of the game theoretic models in infocommunication problems. Our main goal is now to model why "smarter" people win more frequently in some well-known zero-sum games. There are several ways

${ }^{*}$ The publication was supported in part by the TÁMOP-4.2.2C-11/1/KONV-2012-0001 project supported by the European Union, co-financed by the European Social Fund. 
to define smartness. Our definition of smartness is connected to the concept of iterative reasoning and is introduced later on in Section 4.

We focus on modelling players' optimal strategy choices and expected payoffs. These are both stochastic processes given a certain bimatrix game and the level of iterative reasoning according to which players make their decisions.

We constructed a Matlab script that carries out the requested numerical analysis for any simultaneous, two-person bimatrix game. In our paper we present the relating analytical results, describe our concept and recall some numerical results and visualizations.

The rest of the paper is organized as follows. Section 2 gives of a brief overview of game theoretic approaches to infocommunication problems. It also introduces the notion of reasoning. Section 3 recalls some important results from the field of Markov chains that are related to our topic. Section 4 describes our concept of outguessing equilibrium and presents the main theoretical result. Section 5 describes the Matlab script and provides numerical evidence. Finally, Section 6 concludes.

\section{Game theory - infocommunication problems - rea- soning}

There is a numerous amount of topics where game theory is applied. We give some examples (based on $[1,10,16,18,19,20]$. of application of game theory in different infocommunication fields. Identifying and predicting behaviors that are far more likely to be encountered in real-life management circumstances can be handled by reviewing networked games, such as online role-playing games. Such games also provide a good starting point to incorporate network theory into the overarching structure of using game theory to identify the most effective and appropriate set(s) of strategies for effective managerial decision-making. Similarly, when an organization is on the receiving end of significant losses, a more broad-based and intense losing spiral becomes more likely due to the nature of the changing payoff matrices.

By using game theory, one can understand incentives of selfish autonomous agents and large players such as service and content providers. Game-theoretic models are applied in competition for resource allocation among heterogeneous users in wire line and wireless networks. In this case user terminals are players competing for network resources. A few groups of researchers have started advocating the utilization of game theoretic approaches in the network security solutions. As game theory deals with problems where multiple players with contradictory objectives compete with each other, it can provide us with a mathematical framework for analysis and modeling network security problems. As an example, a network administrator and an attacker can be viewed as two competing players participating in a game. In these models game means a description of the strategic interaction between opposing, or cooperating, interests where the constraints and payoff for actions are taken into consideration. Hacker's activities have significantly 
increased in cyberspace, and have been causing damage by exploiting weaknesses in information infrastructure. Recently, researchers have proposed a handful of competing solutions.

In social network analysis game theoretic models are also introduced. In many network settings, the behavior of the system is driven by the actions of a large number of autonomous individuals (or agents). Individuals are self-interested and optimize their respective objectives. Classical game theory predicts how rational agents behave in strategic settings, such as in the domain of advertising, during business interactions, and on the labor market. Although it allows for making predictions regarding human behavior, it makes very strong assumptions. Humans in the real world, on the other hand, are quite different. Their behavior is sometimes emotional, they sometimes base decisions on concepts such as fairness and reciprocity (rather than only on the monetary amount they receive), and are bounded in their reasoning capabilities and thus often use heuristic reasoning. The use of online social networks for behavioral game theory experiments overcomes some of the problems associated with laboratory experiments, and also has some unique benefits.

Almost all the communication systems follow some standards, e.g., the Internet architecture follows the popular TCP/IP protocol suits where all the involved network entities are assumed to follow the rules of the protocol in exact order. However, the cooperation to follow a certain protocol cannot be taken as for granted. This maximization of one's own benefit in a communication system at the cost of other users is called selfishness in game theory. There are some approaches to cope with this selfish behavior in an otherwise cooperative communication system. Tackling this behavior can be found in non-cooperative game theory. This theory gives a perfect match and aim to solve the selfish behavior of the individual entities in a network by outlining rules of the game, which are the same for all the participant agents. By not following these rules of the game, generally, no network entity can be better off while others are following the same set of rules. This gives a huge advantage to address the non-cooperative behavior as far as telecommunication systems are concerned.

Comparatively, designing cooperative games in a large system like the Internet and other scalable networks faces many challenges ranging from efficiency, complexity and fairness among the individual users. The fundamental role of the cooperation among the entities of a network and their effect on the overall system performance has been reported in recent studies.

The phenomenon that human behavior is not purely rational in certain interactive network situations led researchers to construct behavioral game theory models. Recently, several models have been built to explain experimental results (e.g. $[3,8])$.

A popular model class aiming at explaining how players outguess each other is the group of iterative reasoning models. Iterative reasoning has been applied in many settings to the Rock-Paper-Scissors game or the Beauty contest-type games $([4,6,9,13,14,21])$. 
The concept of iterative reasoning and the corresponding main results is presented in [5, pages 205-236]. A simplified concept for non-cooperative, two-person, simultaneous games can be defined as follows. If Player A plays a certain action, while Player B plays the best response to this action, then we say that Player B outguessed Player A and played according to 1-reasoning. If now Player A outguesses Player B, then Player A plays according to 2-reasoning. Following this rule, the level of reasoning can be any $k$ positive integer, where the concept is defined as $k$-reasoning.

Reasoning is the key concept of the present paper.

\section{Markov chains - definitions and some important results}

It is necessary to recall some basic results from the field of Markov chains that we use in the upcoming sections. For a more detailed analysis, we refer the reader to $[7,11,12,17]$. This section is a brief summary of Chapter 4 in [7, pages 119-155], that is related to our concept. The proofs are always omitted.

Definition 3.1. Let $S$ be a countable (finite or countably infinite) set. An $S$ valued random variable $X$ is a function from a sample space $\omega$ into $S$ for which $\{X=x\}$ is an event for every $x \in S$.

Definition 3.2. A matrix $P=(P(i, j))_{i, j \in S}$ with rows and columns indexed by $S$ is called a one-step transition matrix if $P(i, j) \geq 0$ for all $i, j \in S$ and $\sum_{j \in S} P(i, j)=1$ for all $i \in S$.

Definition 3.3. We say that $\left\{X_{n}\right\} n \geq 0$ is a Markov chain in a countable state space $S$ with one-step transition matrix $P$ if $X_{0}, X_{1}, \ldots$ is a sequence of jointly distributed $S$-valued random variables with the property that

$$
P\left(X_{n+1}=j \mid X_{0}, \ldots, X_{n}\right)=P\left(X_{n+1}=j \mid X_{n}\right)=P\left(X_{n}, j\right)
$$

for all $n \geq 0$ and $j \in S$.

We assume that the sequence $X_{0}, X_{1}, \ldots$ is indexed by time, and if we regard time $n$ as the present, the first equation in (3.1), known as the Markov property, says that the conditional distribution of the state of the process one time step into the future, given its present state as well as its past history, depends only on its present state. The second equation in (3.1) tells us that $P\left(X_{n+1}=j \mid X_{n}=i\right)=P(i, j)$ does not depend on $n$. This property is called time homogeneity. The distribution of $X_{0}$ is called the initial distribution $\pi$ and is given by $\pi(i):=P\left(X_{0}=i\right), i \in S$.

A Markov chain can be described by specifying its state space, its initial distribution, and its one-step transition matrix. 
Definition 3.4. We define the m-step transition matrix $P^{m}$ of the Markov chain by

$$
P^{m}(i, j)=\sum_{i_{1}, \ldots, i_{m-1} \in S} \cdots \sum_{P} P\left(i, i_{1}\right) P\left(i_{1}, i_{2}\right) \cdots P\left(i_{m-1}, j\right)
$$

Throughout the paper we apply the following terms ${ }^{1}$ : first hitting time of a state; transient and recurrent states; irreducible Markov chains; stationary distribution; period of state; aperiodic Markov chain.

The following theorem is an important building block in the construction of the outguessing equilibrium (see Section 4):

Theorem 3.5. If an irreducible aperiodic Markov chain in $S$ with one-step transition matrix $P$ has a stationary distribution $\pi$, then it is recurrent and

$$
\lim _{n \rightarrow \infty} P^{n}(i, j)=\pi(j) i, j \in S
$$

Furthermore, $\pi(i)>0$ for all $i \in S$.

Thus, an irreducible aperiodic Markov chain in a finite state space $\mathrm{S}$ has a stationary distribution.

In the following section we define our concept and point out its relationship with Markov chains.

\section{The outguessing equilibrium}

This section presents our notion of outguessing equilibrium. We recall the main assumptions and the key result of the model. For a more detailed overview with examples the reader is referred to [2].

We first define the family of games we analyze.

Definition 4.1. In a two person simultaneous normal form game we denote the players by $i=1,2$. We denote by $S_{i}$ the pure strategy set of player $i$, where $s_{i} \in S_{i}$ and $S=\mathrm{X}_{i=1}^{2} S_{i}$ The utility (or payoff) of any player $i$ is given by $u_{i}\left(s_{i}, s_{-i}\right) \in \mathbb{R}$, where $s_{-i}$ denotes the strategy chosen by the other player.

Our main assumptions are as follows.

Assumption 4.2. We restrict attention to generic games, i.e. where the best response correspondance is a function. That means that there exists only one best response for any action of any of the two players.

Assumption 4.3. The game does not have a pure strategy Nash equilibrium.

Notice that if the game had a pure strategy Nash equilibrium, mixed strategies and probability distributions would not have to be dealt with.

\footnotetext{
${ }^{1}$ Definitions and relating theorems can be found in [7, pages $\left.119-155\right]$
} 
Assumption 4.4. The game is repeated, the rounds are denoted by $1,2, \ldots, n, \ldots$

Assumption 4.5. The players are assumed to keep in mind the strategy profile of the previous round (i.e. their own previous choice and their opponent's previous choice) and nothing else.

Assumption 4.6. Players are assumed to play according to 0-reasoning, 1-reasoning, 2-reasoning ...k-reasoning, or a according to a probability distribution of the different reasoning levels. The distributions are exogenously given and do not change among different rounds of the game.

The definition of the different reasoning levels are discussed in Section 1. Besides, we define 0-reasoning by playing the same strategy as in the previous round.

The exogenously given distribution over the set of reasoning levels is defined as follows.

Definition 4.7. For any player $i$ and any reasoning level $k$ let $P_{i k}$ denote the probability of acting according to $k$-reasoning.

A player is considered smarter than its opponent if his expected reasoning level is higher than that of his opponent. This is how we grab the difference in the complexity of human thinking and try to point out why smarter people may win more frequently in several strategic interactions.

We begin the analysis with the description of the equilibrium concept for the simplest case, where both players have two strategies each.

\subsection{The 2-by-2 model}

Initially, we restrict attention to two-player $2 \times 2$ games with the following general payoff matrix:

\begin{tabular}{lll|cc} 
& & & \multicolumn{2}{|c}{ Player 2 } \\
& & & $q$ & $1-q$ \\
& & & Left & Right \\
\hline \multirow{2}{*}{ Player 1 } & $p$ & Top & $u_{T L} ; v_{T L}$ & $u_{T R} ; v_{T R}$ \\
& $1-p$ & Bottom & $u_{B L} ; v_{B L}$ & $u_{B R} ; v_{B R}$ \\
\hline
\end{tabular}

Table 1: The 2-by-2 game

According to Table 1, Player 1's strategies are Top and Bottom, while Player 2 can choose between Left and Right. $p, 1-p, q, 1-q$ are the respective strategy choice probabilities. Finally, $u_{i j}, v_{i j}$ (where $i \in\{T, B\}$ and $j \in\{L, R\}$ ) are the two players' payoff levels given a certain strategy pair.

According to Assumption 4.3, we assume that the game does not have a pure strategy Nash-equilibrium. A necessary and sufficient condition for this is

$$
u_{T L}>u_{B L}, u_{B R}>u_{T R}, v_{T R}>v_{T L}, v_{B L}>v_{B R} .
$$


This means that the best responses of both players are given for any action of their opponent. E.g. if Player 1 chooses Top, then Player 2's best response is Right, as $v_{T R}>v_{T L}$.

We introduce our concept of play history in the next definition.

Definition 4.8. We use the notion history for the strategy profile of the previous round of the game.

The history of the game described by Table 1 can be the following: (Top;Left), (Top;Right), (Bottom;Left) and (Bottom;Right).

Depending on the history, we can define four different games, where the strategies and the payoffs are the same. The only difference is that both players keep the history in mind and this has an influence on their decisions, i.e. their strategy mixing probabilities.

The payoff and probability matrices with the (Top, Left) history is as follows. The other three versions can be constructed the same way, with TR, BL and BR indices, respectively.

\begin{tabular}{|c|c|c|c|c|}
\hline & & & \multicolumn{2}{|c|}{ Player 2} \\
\hline & & & $\begin{array}{l}q_{T L} \\
\text { Left }\end{array}$ & $\begin{array}{c}1-q_{T L} \\
\text { Right }\end{array}$ \\
\hline Player 1 & $\begin{array}{l}p_{T L} \\
1-p_{T L}\end{array}$ & $\begin{array}{l}\text { Top } \\
\text { Bottom }\end{array}$ & $\begin{array}{l}u_{T L} ; v_{T L} \\
u_{B L} ; v_{B L}\end{array}$ & $\begin{array}{l}u_{T R} ; v_{T R} \\
u_{B R} ; v_{B R}\end{array}$ \\
\hline
\end{tabular}

Table 2: The game with (Top, Left) history

If we consider any 2-by-2 game that satisfies our assumptions and a first-round strategy profile and a distribution on the set of reasoning levels is given for both players, there emerge the following questions:

1. What is the ex ante strategy choice distribution of the two players if the number of rounds $n \rightarrow \infty$ ? Is there any limiting distribution?

2. What is the expected payoff of the players for each round if $n \rightarrow \infty$ ? Is the series of expected payoffs convergent?

To answer the questions, we will apply the theory of Markov chains.

\subsection{The Markov chain model of the 2-by-2 game}

The outguessing model can be interpreted as a Markov chain. According to Assumption 4.5, the players keep in mind only the actions of the previous round of the game. Let us define the Markov chain of the described 2-by-2 game.

Proposition 4.9. The strategy profile sequence of the repeated game represents a Markov chain. 
Proof. The proof comes directly from Definition 3.3 and Assumption 4.5 that show that the strategy profile sequence $\left\{X_{n}\right\} \quad(n \geq 1)$ has the Markov property.

In a 2-by-2 game we have 4 different strategy profiles, in our example these are (Top;Left), (Top;Right), (Bottom;Left) and (Bottom;Right), or in a shorter form: TL, TR, BL, BR. Thus, we can define the four states as follows:

\begin{tabular}{cc}
\hline \hline State no. & Strategy profile \\
\hline 1 & (Top;Left) \\
2 & (Top;Right) \\
3 & (Bottom;Left) \\
4 & (Bottom;Right) \\
\hline
\end{tabular}

Table 3: States of the Markov chain

The transition matrix of the Markov chain can be obtained by using the data of the general payoff and probability matrices from the previous subsection.

Proposition 4.10. The 4-by-4 transition matrix can be written as follows:

$$
T=\left(\begin{array}{cccc}
p_{T L} q_{T L} & p_{T L}\left(1-q_{T L}\right) & \left(1-p_{T L}\right) q_{T L} & \left(1-p_{T L}\right)\left(1-q_{T L}\right) \\
p_{T R} q_{T R} & p_{T R}\left(1-q_{T R}\right) & \left(1-p_{T R}\right) q_{T R} & \left(1-p_{T R}\right)\left(1-q_{T R}\right) \\
p_{B L} q_{B L} & p_{B L}\left(1-q_{B L}\right) & \left(1-p_{B L}\right) q_{B L} & \left(1-p_{B L}\right)\left(1-q_{B L}\right) \\
p_{B R} q_{B R} & p_{B R}\left(1-q_{B R}\right) & \left(1-p_{B R}\right) q_{B R} & \left(1-p_{B R}\right)\left(1-q_{B R}\right)
\end{array}\right)
$$

Proof. The elements of the transition matrix are the probabilities of getting into a given state from a given previous state, i.e. the probabilities that a certain strategy profile will emerge given the strategy profile of the previous round. Using the previously defined $p_{i j}$ and $q_{i j}$ probabilities, and knowing that strategic decisions are independent from each other in a simultaneous game, we obtain the formula in the statement.

Clearly, the transition matrix depends directly only on the players' probabilities of choosing a certain strategy with a given history. The transition matrix is independent from the construction of these probabilities. Thus, it remains the same for all models where the players' actions depend only on the previous round and a probability distribution is exogenously given for both players on the set of reasoning levels.

The following lemma indicates that only 0,1,2 and 3-reasoning levels are relevant for a given 2-by-2 game.

Lemma 4.11. For 2-by-2 games and for all $k \geq 4, k$-reasoning is equivalent to $(k-4)$-reasoning.

Proof. The proof comes directly from the inequalities in (12). 
We need one more definition to be able to state the key result of the paper.

Definition 4.12. Let us denote the initial strategy distribution of the players by $\pi_{0}$.

The distribution over the state space (the set of strategy pairs) in the $n$th round can obviously be calculated as follows:

$$
\pi_{n}=T^{n} \pi_{0}
$$

The key result states that under certain conditions there exists a limiting distribution if $n \rightarrow \infty$.

Proposition 4.13. If $P_{i k}>0$ (see Definition 4.7) for every $i \in\{1,2\}$ and every $k \in\{0,1,2,3\}$ and if $n \rightarrow \infty$, then there exists a limiting distribution $\pi$ over the state space of the $\left\{X_{n}\right\}(n \geq 1)$ Markov chain.

Proof. If $P_{i k}>0$ for every $i \in\{1,2\}$ and every $k \in\{0,1,2,3\}$, then it can easily be verified according to Definition 4.1 that $\left\{X_{n}\right\}$ is an irreducible aperiodic Markov chain. Thus, according to Theorem 11 it is recurrent and has a limiting distribution.

We arrived at our equilibrium concept. The outguessing equilibrium is defined as the limiting distribution.

Definition 4.14. We call the limiting distribution $\pi$ the outguessing equilibrium.

According to the proof of Proposition 4.13, the key result is supported by Theorem 3.5: the strategy profile sequence of the repeated 2-by-2 game represents a Markov chain that has a limiting distribution.

As far as the players' expected payoffs are concerned, they can easily be determined by multiplying $\pi^{\prime}$ ( $\pi$ vector transposed) with the vector of the corresponding payoff levels.

By running our script, the limiting distribution $\pi$ and the long-term expected payoffs can be calculated and visualized. It becomes clear that the player with the higher expected reasoning level has the higher expected payoff on the long run.

\section{Numerical experiments}

\subsection{Description of the script}

Our script was written in Matlab. Its inputs are the following values: the payoff matrix of the corresponding 2-by-2 or 3-by-3 game; the players' discrete probability distributions over the set of reasoning levels; initial strategies (i.e. player behavior in the very first round - either a fixed strategy pair or an initial distribution).

The script works the following way. Firstly, from the given values it calculates the transition matrix of the Markov chain. Then, the outguessing equilibrium 
(see Definition 4.1) and the long-term expected payoffs for both players are also calculated (based on several repetitions of the game). Proposition 4.13 suggests that the "smarter" player (if there is one) beats its opponent on the long run.

The simplified pseudocode of the script is as follows:

\section{Algorithm 5.1: Outguessing Equilibrium (Matlab)}

$M \leftarrow$ Payoff matrix values

$P_{i} \leftarrow$ Player i's distribution over the reasoning levels $(\forall i) S_{0}$ $\leftarrow$ Initial strategy profile distribution

$E_{0} \leftarrow$ Initial expected payoff vector

do $T$ (calculation of the transition matrix)

for $r \leftarrow 0$ to 100

do $S_{r}$ (the strategy profile distribution for the rth round)

do $E_{r}$ (the expected payoff vector for the rth round)

The power of the script is that the outguessing equilibrium concept can be tested for any 2-by-2 or 3-by-3 bimatrix game that does not have a Nash equilibrium on pure strategies. ${ }^{2}$

\subsection{An example: the matching pennies}

In the matching pennies game, both players have to announce "heads" or "tails" at the same time. If the announcements are the same, Player 1 wins 1 from Player 2, otherwise Player 2 wins 1 from Player 1 . The payoff matrix of the well-known zero-sum game is depicted in Table 4:

\begin{tabular}{lll|cc} 
& & & \multicolumn{2}{|c}{ Player 2 } \\
& & & $q$ & $1-q$ \\
& & & Left & Right \\
\hline \multirow{2}{*}{ Player 1 } & $p$ & Top & $1 ;-1$ & $-1 ; 1$ \\
& $1-p$ & Bottom & $-1 ; 1$ & $1 ;-1$ \\
\hline
\end{tabular}

Table 4: Payoff matrix of the Matching Pennies game

The Nash-equilibrium mixing probabilities are 50\%-50\% for both players.

Let us assume that in our model the initial strategy choice probabilities are 0.5 each (in the first round when there is no history). For the distributions over the set of reasoning levels (see Definition 14) let us assume that $P_{10}=0.4, P_{11}=0.2$, $P_{12}=0.2, P_{13}=0.2$, while $P_{10}=0.2, P_{10}=0.2, P_{10}=0.4, P_{10}=0.4$. Clearly, Player 2 is considered smarter due to his higher expected reasoning level.

We ran our script and the process of the expected payoffs calculated from $\left(\pi_{1}, \pi_{2}, \ldots, \pi_{n}, \ldots\right)$. The results predict that these processes converge to certain limit

\footnotetext{
${ }^{2}$ Upon request, the authors provide the interested reader with the script for testing purposes.
} 
values, a numerical evidence for Proposition 4.13. It is also verified for the Matching pennies that the smarter Player 2 (represented by the above "+" sequence) has a higher expected payoff (0.232) than Player 1 (-0.232) on the long run. According to the mixed strategy Nash equilibrium concept, both players would have zero expected payoff.

One could alter the following input arguments: the elements of the payoff matrix; the distribution over the players' reasoning levels; the initial strategy choice of the two players.

However, as Proposition 4.13 suggests, the key result remains valid for every game variant that does not have a pure Nash equlibrium. The only difference is the speed of convergence to the outguessing equilibrium. A typical plot of the long-term expected payoffs for a slow-convergence case is depicted in Figure 1.

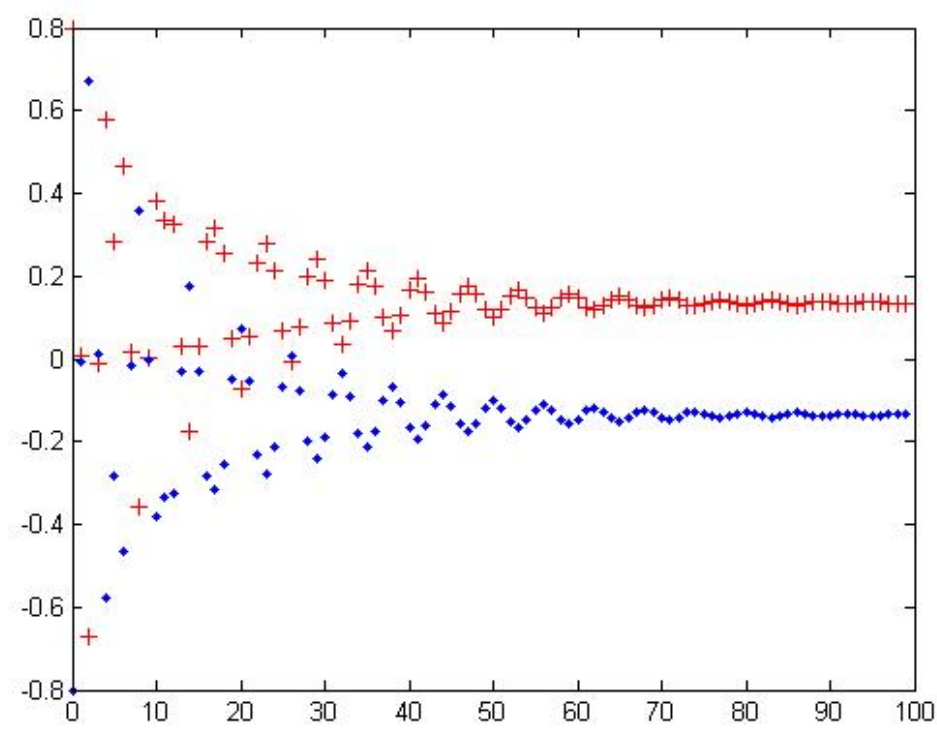

Figure 1: A typical plot of long-term expected payoffs depending on the number of rounds

\section{Conclusion}

In the present paper we have developed a behavioral model that aims at showing why "smarter" people win more often in certain bimatrix games.

We used the concept of iterative reasoning to define smartness. We also made use of the theory of Markov chains to prove the main result of the paper, i.e. 
an outguessing equilibrium according to our definition always exists and can be calculated by numerical tools.

It can be shown that independently from the input data, the stochastic processes of the players' long-term expected payoffs always converge to a limiting distribution that is called the outguessing equilibrium. A Matlab script supports the calculations and provides numerical evidence.

The presented model can not only be applied for the games recalled in the example, but for any conflict situation that can be modeled by bimatrix games.

\section{References}

[1] Alpcan, T., Basar, T., A Globally Stable Adaptive Congestion Control Scheme for Internet-Style Networks with Delay, IEEE/ACM Trans. On Networking Vol. 13 (2005), 1261-1274.

[2] Balogh T. L., Kormos J., A computational model of outguessing in two-player non-cooperative games, Acta Informatica Vol. 6 (2014), 71-88, forthcoming

[3] BeARD, S.C., Beil, R., Do people rely on the self-interested maximization of others? An experimental test, Management Science Vol. 40 (1994), 252-262.

[4] Cabrera, S.C., Capra, C.M., Gomez, R., The effects of common advice on oneshot traveller's dilemma games: Explaining behavior through an introspective model with errors, Washington and Lee University working paper, 2002.

[5] Camerer, C.F., Behavioral Game Theory: Experiments in Strategic Interaction, Princeton University Press, 2003, pp. 199-264.

[6] Capra, C. M., Goeree, J.K., Gomez, R., Holt, C., Anomalous behavior in traveller's dilemma?, American Economic Review, Vol. 89 (1999), 678-690.

[7] S.N. Ethier, The Doctrine of Chances, Springer, 2010, pp. 119-155.

[8] Goeree, J.K., Holt, C., Stochastic game theory: For playing games, not just for doing theory, Proceedings of the National Academy of Sciences 96 (1999), 1056410567.

[9] Ho, T., Camerer, C.F., Weigelt, K. Iterated dominance and iterated bestresponse in experimental "p-beauty contests", American Economic Review, Vol. 88 (1998), 947-969.

[10] Krishnamurthy V., Zhao, Q., Wen, M. (Eds.), Game Theory for Networks, Springer, 2012.

[11] Meyn, S.P., Tweedie, R.L., Markov chains and stochastic stability, Cambridge University Press, 2009.

[12] Meyn, S.P., Control Techniques for Complex Networks, Cambridge University Press, 2007.

[13] Moulin, Game Theory for the Social Sciences, New York University Press, 1986.

[14] Nagel, R. Unravelling in guessing games: an experimental study, American Economic Review, Vol. 85 (1995), 1313-1326.

[15] NAsh, J. Non-cooperative games, Annals of Mathematics (1951), 286-295. 
[16] Narayanam, R., Game Theoretic Models for Social Network Analysis, Working Paper, 2012.

[17] Norris, J.R., Markov chains, Cambridge University Press, 1998.

[18] Ozdaglar, A., Networks' Challenge: Where Game Theory Meets network Optimization, International Symposium on Information Theory, MIT, 2008.

[19] Roy, S., Ellis, C., Shiva, S., Dasgupta, D., Shandilya, Q., Wu, Q., A Survey of Game Theory as Applied to Network Security, http://www.computer.org/csdl/proceedings/hicss/2010/3869/00/02-02-03abs.html, 2010.

[20] Shah, I.A., Jan, S., Khan, I., Qamar, S., An overview of game theory and its applications in communication networks, International Journal of Multidisciplinary Sciences and Engineering, Vol. 3 (2012), 5-11.

[21] Sovik, Y., Impossible bets: an experimental study, University of Oslo working paper, 1999. 